Pacific Journal of Mathematics

COUNTING FUNCTIONS AND MAJORIZATION FOR JENSEN 


\title{
COUNTING FUNCTIONS AND MAJORIZATION FOR JENSEN MEASURES
}

\section{Charles S. Stanton}

\begin{abstract}
We establish a generalization for uniform algebras of the classical identities of Hardy and Stein. We use this and an estimate based on the isoperimetric inequality to give a proof of $\mathbf{H}$. Alexander's spectral area theorem. We use similar methods to prove a theorem of Axler and Shapiro about VMOA of the unit ball in $\mathrm{C}^{n}$.
\end{abstract}

1. Introduction. Given a Jensen measure on the maximal ideal space of $A$, we introduce a "counting function" analogous to the classical counting function $N(r, w)$ of Nevanlinna's value distribution theory. In particular, this counting function is non-negative, supported on the spectrum of $f$, and a subharmonic function of $w$ on the complex plane except for a logarithmic pole. We next establish an identity for integral means of $f$ in terms of this counting function. This generalizes Theorems 2 and 9 of [6]. Classical identities of Cartan and of Hardy and Stein occur as special cases.

As an application, we give a proof (for Jensen measures) of $\mathrm{H}$. Alexander's spectral area estimate:

TheOrem A $[1,2]$. Let $A$ be a uniform algebra, $\varphi \in M_{A}$, and $\sigma$ a Jensen measure for $\varphi$. Then

$$
\int_{M_{A}}|f|^{2} d \sigma \leq \frac{1}{\pi} \operatorname{area}(\operatorname{spec} f)+|f(\varphi)|^{2}
$$

Finally, we apply these counting function techniques to prove a slight generalization of the following result of Axler and Shapiro about analytic functions of vanishing mean oscillation (VMOA) of the unit ball in $\mathbf{C}^{n}$.

TheOrem B [3]. Suppose $f \in H^{\infty}\left(\mathbf{B}^{n}\right)$ and for each $\zeta \in S$

$$
\operatorname{area}(\operatorname{cl}(f, \zeta))=0 \text {. }
$$

Then $f \in$ VMOA.

2. Uniform algebras and Jensen measures. We first recall some basic facts about uniform algebras and Jensen measures (for more details 
see [7]). We then introduce the counting function and establish its subharmonicity.

Let $X$ be a compact Hausdorff space and $A$ a uniform algebra on $X$, i.e. a closed subalgebra of $C(X)$ which contains the constants and separates points of $X$. Let $M_{A}$ denote the maximal ideal space of $A$. The spectrum of $f \in A$, denoted $\operatorname{spec} f$, is the set $\{w \in \mathbf{C}: f-w$ is not invertible in $A$ \}.

Let $\varphi \in M_{A}$. A probability measure $\sigma$ on $M_{A}$ is a Jensen measure for $\varphi$ if and only if

$$
\log |f(\varphi)| \leq \int_{M_{A}} \log |f| d \sigma,
$$

for every $f \in A$. Since $\sigma$ is a Jensen measure it is also an Arens-Singer measure for $\varphi$ :

$$
\log |f(\varphi)|=\int_{M_{A}} \log |f| d \sigma,
$$

for each invertible $f$ belonging to $A$. It follows that $\sigma$ is also a representing measure for $\varphi$ :

$$
f(\varphi)=\int_{M_{A}} f d \sigma
$$

for each $f \in A$.

Definition. Suppose $f \in A, \varphi \in M_{A}$, and that $\sigma$ is a Jensen measure for $\varphi$. Then, for each $w \in \mathbf{C} \backslash\{f(\varphi)\}$, we define

$$
N(w ; f, \sigma)=\int_{M_{A}} \log |f-w| d \sigma-\log |f(\varphi)-w| .
$$

REMARK. If $\sigma=\alpha \tau+(1-\alpha) \delta_{\varphi}$ with $0<\alpha<1$ then $\tau$ is also a Jensen measure for $\varphi$ and $N(w ; f, \sigma)=\alpha N(w ; f, \tau)$. We shall assume that $\sigma(\varphi)=0$ in the following. We shall also denote $N(w ; f, \sigma)$ by $N(w)$ when $f$ and $\sigma$ have been fixed.

The properties of $N(w)$ are summarized by

Proposition 1. Suppose $f \in A, \varphi \in M_{A}$, and that $\sigma$ is a Jensen measure for $\varphi$. Then $N(w)$ is a non-negative function supported on spec $f$. Furthermore, $N(w)$ is subharmonic on $\mathbf{C} \backslash\{f(\varphi)\}$, and $N(w)+$ $\log |f(\varphi)-w|$ is subharmonic on $\mathbf{C}$.

Proof. The non-negativity is a consequence of the definition (2) of a Jensen measure; since a Jensen measure is also an Arens-Singer measure 
the support of $N(w)$ is contained in $\operatorname{spec} f$ by (3). To prove the subharmonicity we introduce the Borel probability measure $f^{*}(d \sigma)$ supported on $\operatorname{spec} f$ defined by

$$
\int_{M_{A}}(k \circ f) d \sigma=\int_{\mathbf{C}} h(\zeta) f^{*} d \sigma(\zeta)
$$

for every $h \in L^{1}(d \sigma)$. Thus

$$
\int_{M_{A}} \log |f-w| d \sigma=\int_{\mathbf{C}} \log |\zeta-w| f^{*}(d \sigma) .
$$

This establishes $N(w)+\log |f(\varphi)-w|$ as the potential of the measure $f^{*}(d \sigma)$ and hence a subharmonic function on C. Since $\log |f(\varphi)-w|$ is harmonic on $\mathbf{C} \backslash\{f(\varphi)\}$, we set that $N(w)$ is subharmonic on $\mathbf{C} \backslash\{f(\varphi)\}$.

When $A$ is the disc algebra the following result is known as Lehto's principle of majorization (see [10]):

THEOREM 1. Let $\Omega$ be an open set which contains $\operatorname{spec} f$, and $G_{\Omega}(w ; f(\varphi))$ be the Green function for $\Omega$ with pole at $f(\varphi)$. Then

$$
N(w) \leq G_{\Omega}(w ; f(\varphi)) .
$$

REMARK. We shall always extend a Green function $G_{\Omega}$ to all of $\mathbf{C}$ by defining it to be identically zero outside of $\Omega$.

Proof. The theorem follows immediately from the maximum principle since $G_{\Omega}(w ; f(\varphi))+\log |f(\varphi)-w|$ is harmonic on $\Omega$ while $N(w)+$ $\log |f(\varphi)-w|$ is subharmonic on $\Omega$ and $N(w) \leq G_{\Omega}(w ; f(\varphi))$ on the boundary of $\Omega$.

3. Identities for integral means. Our next result expresses integral means of $f$ as an integral of $N(w)$ weighted by an appropriate measure.

Theorem 2. Suppose $\Psi$ is subharmonic on a disc $\Delta_{R}=\{z:|z|<R\}$ which contains $\operatorname{spec} f$. Let $d \mu$ be the Riesz measure for $\Psi$. Assume $\mu(f(\varphi))=0$. Then

$$
\int_{M_{A}} \Psi(f) d \sigma=\int_{\mathbf{C}} N(w) d \mu+\Psi(f(\varphi)) .
$$

Proof. By the Riesz decomposition theorem for subharmonic functions

$$
\Psi(\zeta)=\int_{\text {spec } f} \log |w-\zeta| d \mu(w)+h(\zeta)
$$


Here the Riesz measure $d \mu=(1 / 2 \pi) \Delta \Psi$ in the sense of distributions and $h$ is harmonic in the interior of $\Delta_{R}$. Thus $h=\operatorname{Re} H$ for some function $H$ holomorphic on $\{w:|w|<R\}$. It follows from the "functional calculus" that $H \circ f \in A$. Since $\sigma$ is a representing measure for $\varphi$ we have by (4)

$$
\int_{M_{A}} h \circ f d \sigma=h(f(\varphi)) .
$$

We now calculate, using the Riesz decomposition (8) and the definition of $N(w)$ :

$$
\begin{aligned}
\int_{M_{A}} \Psi \circ f d \sigma & =\int_{M_{A}}\left\{\int_{\Delta_{R}} \log |w-f| d \mu(w)+h \circ f\right\} d \sigma \\
& =\int_{\Delta_{R}} \int_{M_{A}} \log |w-f| d \sigma d \mu(w)+h(f(\varphi)) \\
& =\int_{\Delta_{R}} N(w)+\log |f(\varphi)-w| d \mu(w)+h(f(\varphi)) \\
& =\int_{\Delta_{R}} N(w) d \mu(w)+\Psi(f(\varphi)) .
\end{aligned}
$$

Since $N(w)$ is supported on $\operatorname{spec} f$ we may extend the last integral to be taken over the entire plane to obtain (7).

Two important special cases of (7) occur when we take $\Psi(\zeta)=\log ^{+}|\zeta|$ and $\Psi(\zeta)=|\zeta|^{p}$. In the first case Theorem 2 implies

$$
\int_{M_{A}} \log ^{+}|f| d \sigma=\frac{1}{2 \pi} \int_{0}^{2 \pi} N\left(e^{i \vartheta}\right) d \vartheta+\log ^{+}|f(\vartheta)| .
$$

If $A$ is the disc algebra and $d \sigma$ is Lebesgue measure on the unit circle this is known as Cartan's formula [9, p. 8]. In the second case we obtain, for $p>0$,

$$
\int_{M_{A}}|f|^{p} d \sigma=\frac{p^{2}}{2 \pi} \int_{\mathbf{C}} N(w)|w|^{p-2} d u d v+|f(\varphi)|^{p} .
$$

which is a version of the Hardy-Stein identity [13]. For applications of other choices of $\Psi$ see [6].

4. Alexander's spectral area theorem. The key estimate we will need is the following consequence of the isoperimetric inequality:

Proposition 3 ([11, p. 115], [4, p. 60]). Let $\Omega$ be a plane domain of finite area. Let $G_{\Omega}\left(w, w_{0}\right)$ be the Green function for $\Omega$ with pole at $w_{0}$. Then

$$
\int_{\Omega} G_{\Omega}\left(w, w_{0}\right) d u d v \leq \frac{1}{2} \operatorname{area}(\Omega) .
$$


Proof of Theorem A. Let $\Omega$ be a region containing spec $f$ such that area $\Omega \leq \operatorname{area}(\operatorname{spec} f)+\varepsilon$. By the Hardy-Stein identity (10), Lehto's principle of majorization (6), and the proposition above we have

$$
\begin{aligned}
\int_{M_{A}}|f|^{2} d \sigma & =\frac{2}{\pi} \int_{\mathbf{C}} N(w) d u d v+|f(\varphi)|^{2} \\
& \leq \frac{2}{\pi} \int_{\mathbf{C}} G_{\Omega}(w ; f, \varphi) d u d v+|f(\varphi)|^{2} \\
& \leq \frac{2}{\pi} \frac{1}{2} \operatorname{area}(\Omega)+|f(\varphi)|^{2} \\
& \leq \frac{1}{\pi}(\operatorname{area}(\operatorname{spec} f)+\varepsilon)+|f(\varphi)|^{2} .
\end{aligned}
$$

Letting $\varepsilon \rightarrow 0$ we obtain (1).

5. Counting functions on $\mathbf{B}^{n}$. Let $\mathbf{B}^{n}$ denote the open unit ball in $\mathbf{C}^{n}$ with normalized measure $d \sigma$ on $\partial \mathbf{B}^{n}$. Suppose $\alpha \in \mathbf{B}^{n}$. The Poisson-Szegö measure for $\alpha$ is

$$
d \nu_{\alpha}(\zeta)=\left\{\frac{1-|\alpha|^{2}}{|1-\langle\alpha, \zeta\rangle|^{2}}\right\}^{n} d \sigma(\zeta) .
$$

The Möbius transformation $\varphi_{\alpha}$ is defined by

$$
\varphi_{\alpha}(z)=\frac{\alpha-P_{\alpha} z-\left(1-|\alpha|^{2}\right)^{1 / 2} Q_{\alpha} z}{1-\langle z, \alpha\rangle},
$$

where $P_{\alpha}$ is the orthogonal projection of $\mathbf{C}^{n}$ onto the subspace generated by $\alpha$, and $Q_{\alpha}$ is the orthogonal complement to $P_{\alpha}$. The properties of $\varphi_{\alpha}$ are summarized by

Proposition 4. Let $\alpha \in \mathbf{B}^{n}, z \in \overline{\mathbf{B}^{n}}, h \in \mathbf{C}^{n}$, and $g \in L^{1}\left(\partial \mathbf{B}^{n}\right)$. Then

(12.d) $\varphi_{\alpha}(z) h=\frac{\left(1-|\alpha|^{2}\right)^{1 / 2}}{(1-\langle z, \alpha\rangle)^{2}}$

$$
\times\left\{-\left(1-|\alpha|^{2}\right)^{1 / 2} P_{\alpha} h-Q_{\alpha} h-z\langle h, \alpha\rangle+h\langle z, \alpha\rangle\right\}
$$




$$
\int_{\partial \mathbf{B}^{n}} g \circ \varphi_{\alpha} d \sigma=\int_{\partial \mathbf{B}^{n}} g d \nu_{\alpha}
$$

Proof. Assertions (12.a-12.c) are contained in Theorem 2.2.2 of [12], and (12.d) may be obtained in the same manner as part (ii) of that Theorem. Part (12.e) is Theorem 3.3.8 of [12].

The Green function with pole at 0 for $\mathbf{B}^{n}$ is

$$
G_{\mathbf{B}}(z, 0)= \begin{cases}\log \frac{1}{|z|} & (\text { if } n=1) \\ \frac{1}{2 n-2}\left(|z|^{2-2 n}-1\right), & (\text { if } n>1)\end{cases}
$$

We introduce the form

$$
\beta=\frac{i}{2 \pi} \sum_{j} d z_{j} \wedge d \bar{z}_{j}
$$

Then $\beta^{n}$ is Lebesgue measure on $\mathbf{C}^{n}$, normalized so that $\int_{\mathbf{B}} \beta^{n}=1$. We recall Wirtinger's Theorem [8, p. 5]: If $V$ is a $k$-dimensional variety then $\beta^{k}$ is the induced volume form.

It follows from Jensen's formula [14, p. 248] that $d \sigma$ is a Jensen measure for 0 and that the counting function $N(0 ; f, \sigma)$ defined by $(5)$ is the usual counting function of value distribution theory in $\mathbf{C}^{n}$. In particular, if $\mu(z)$ is the multiplicity of the zero at $z$ then $[14$, p. 248]

$$
N(0 ; f, \boldsymbol{\sigma})=\int_{f^{-1}(0)} \mu(z) G_{\mathbf{B}}(z, 0) \beta^{n-1} .
$$

It follows from (5) and (12.e) that

$$
N\left(w ; f, \nu_{\alpha}\right)=N\left(0 ; f \circ \varphi_{\alpha}-w, \sigma\right)
$$

and hence

$$
N\left(w ; f, \nu_{\alpha}\right)=\int_{\varphi_{\alpha}\left(f^{-1}(w)\right)} \mu(z) G_{\mathbf{B}}(z, 0) \beta^{n-1}
$$

where $\mu$ is the multiplicity of the zero for $f \circ \varphi_{\alpha}-w$ at $z$. Since the integrand on the right is non-negative it follows that $\nu_{\alpha}$ is a Jensen measure for $\alpha$.

The counting function $N\left(w ; f, \nu_{\alpha}\right)$ may be extended to $f$ in the Nevanlinna class (see [5, \$4] for more details) by setting

$$
N\left(w ; f, \nu_{\alpha}\right)=\limsup _{\zeta \rightarrow w}\left(\lim _{r \rightarrow 1}\left(N\left(w ; f_{r}, \nu_{\alpha}\right)\right)\right)
$$


where $f_{r}(z)=f(r z)$ for $r<1$. Theorem 1 remains true in this context. If $\Psi$ is a positive subharmonic function then Theorem 2 may be extended to

$$
\lim _{r \rightarrow 1} \int_{\partial \mathbf{B}^{n}} f_{r} d \nu_{\alpha}=\int_{\mathbf{C}} N\left(w ; f, \nu_{\alpha}\right) d \mu+\Psi(f(\alpha)) .
$$

6. Functions of vanishing mean oscillation on $\mathbf{B}^{n}$. Definition. A function $f \in H^{2}\left(\mathbf{B}^{n}\right)$ is said to belong to BMOA if

$$
\|f\|_{*}^{2}=\sup _{\alpha \in \mathbf{B}} \int_{\partial \mathbf{B}^{n}}|f-f(\alpha)|^{2} d \nu_{\alpha}
$$

is finite in which case $\|f\|_{*}+|f(0)|$ is a norm on the space BMOA.

Definition. A function in $H^{2}\left(\mathbf{B}^{n}\right)$ belongs to VMOA if for every $\zeta \in \partial \mathbf{B}^{n}$

$$
\lim _{\alpha \rightarrow \zeta} \int_{\partial \mathbf{B}^{n}}|f-f(\alpha)|^{2} d \nu_{\alpha}=0
$$

We note that

$$
\begin{aligned}
\int_{\partial \mathbf{B}^{n}}|f-f(\alpha)|^{2} d \nu_{\alpha} & =\int_{\partial \mathbf{B}^{n}}|f|^{2} d \nu_{\alpha}-|f(\alpha)|^{2} \\
& =\int_{\partial \mathbf{B}^{n}}\left|f \circ \varphi_{\alpha}\right|^{2} d \sigma-|f(\alpha)|^{2} .
\end{aligned}
$$

Since area $\left(f \circ \varphi_{\alpha}\left(\mathbf{B}^{n}\right)\right)=\operatorname{area}\left(f\left(\mathbf{B}^{n}\right)\right)$ it follows from Theorem $\mathbf{A}$ that if area $\left(f\left(\mathbf{B}^{n}\right)\right)$ is finite then $f \in$ BMOA and

$$
\|f\|_{*}^{2} \leq \frac{1}{\pi} \operatorname{area}\left(f\left(\mathbf{B}^{n}\right)\right) \text {. }
$$

For $\zeta \in \partial \mathbf{B}^{n}$ we define

$$
D_{\rho, \zeta}=\left\{z \in \mathbf{B}^{n}:|1-\langle z, \zeta\rangle|<\rho\right\} .
$$

Our generalization of Theorem B is

THEOREM 3. Suppose $f$ is holomorphic in $\mathbf{B}^{n}$ and for every $\zeta \in \partial \mathbf{B}^{n}$

$$
\lim _{\rho \rightarrow 0} \operatorname{area}\left(f\left(D_{\rho, \zeta}\right)\right)=0 \text {. }
$$

Then $f \in$ VMOA.

Before giving the proof of Theorem 3 we will show how Theorem B follows from it. Since the sets $D_{\rho, \zeta}$ form a basis for the topology at $\zeta$ the cluster set of $f$ at $\zeta$ may be defined by

$$
\operatorname{cl}(f, \zeta)=\bigcap_{\rho>0} \overline{f\left(D_{\rho, \zeta}\right)}
$$


We have

$$
\begin{aligned}
\lim _{\rho \rightarrow 0} \operatorname{area}\left(f\left(D_{\rho, \zeta}\right)\right) & =\operatorname{area}\left(\bigcap_{\rho>0} f\left(D_{\rho, \zeta}\right)\right) \\
& \leq \operatorname{area}\left(\bigcap_{\rho>0} \overline{f\left(D_{\rho, \zeta}\right)}\right)=\operatorname{area}(\operatorname{cl}(f, \zeta)) .
\end{aligned}
$$

By the hypothesis of Theorem B area $(\operatorname{cl}(f, \zeta))=0$ so the hypothesis (16) of Theorem 3 is satisfied and hence $f \in$ VMOA.

The equality in the first line of (17) follows from the dominated convergence theorem; we note that the hypothesis $f \in H^{\infty}$ could be replaced by the assumption $\operatorname{area}\left(f\left(\mathbf{B}^{n}\right)\right)$ is finite.

The following two lemmas will be used in the proof of Theorem 3.

Lemma 1 (see [12, Proposition 5.1.2]). If $\alpha, z, \zeta \in \overline{\mathbf{B}^{n}}$ then

$$
|1-\langle z, \zeta\rangle|^{1 / 2}+|1-\langle z, \alpha\rangle|^{1 / 2} \geq|1-\langle\zeta, \alpha\rangle|^{1 / 2} \text {. }
$$

Lemma 2. Suppose $\alpha \in D_{\tau, \zeta}$ with $\tau<\rho / 16$ and $w \notin f\left(D_{\rho, \zeta}\right)$. Then there is a constant $C$ depending only on $\rho$ and $n$ such that

$$
N\left(w ; f, \nu_{\alpha}\right)<C\left(1-|\alpha|^{2}\right)^{n} N(w ; f, \sigma) .
$$

Proof of Lemma 2. Suppose $\alpha \in D_{\tau, \zeta}$ and $z \in \mathbf{B}^{n} \backslash D_{\rho, \zeta}$. We deduce from (18) that $|1-\langle\alpha, z\rangle|>9 / 16$. Hence, by (12.b),

$$
1-\left|\varphi_{\alpha}(z)\right|^{2}=\frac{\left(1-|\alpha|^{2}\right)\left(1-|z|^{2}\right)}{|1-\langle\alpha, z\rangle|^{2}}<\frac{2}{9} .
$$

This implies that $\left|\varphi_{\alpha}(z)\right|>7 / 9$, and thus

$$
G_{\mathbf{B}}\left(\varphi_{\alpha}(z), 0\right) \leq c\left(1-\left|\varphi_{\alpha}(z)\right|\right)^{2}
$$

for some constant $c$ depending only on the dimension $n$.

Now suppose $w \notin f\left(D_{\rho, \zeta}\right)$. Then by (20) and a change of variables

$$
\begin{aligned}
N\left(w ; f, \nu_{\alpha}\right) & =\int_{\varphi_{\alpha}\left(f^{-1}(w)\right)} G_{\mathbf{B}}(z, 0) \beta^{n-1} \\
& =\int_{f^{-1}(w)} G_{\mathbf{B}}\left(\varphi_{\alpha}(z), 0\right) \varphi_{\alpha}^{*} \beta^{n-1} \\
& \leq c \int_{f^{-1}(w)} 1-\left|\varphi_{\alpha}(z)\right|^{2} \varphi_{\alpha}^{*} \beta^{n-1},
\end{aligned}
$$

since $z \in \varphi_{\alpha}\left(f^{-1}(w)\right)$ implies $z \notin D_{\rho, \zeta}$. 
From (12.d) we deduce the estimate

$$
\varphi_{\alpha}^{*} \beta^{n-1} \leq \sup _{|I|=|J|=n-1}\left|\frac{\partial^{I} \varphi_{\alpha}}{\partial z^{J}}\right|^{2} \beta^{n-1} \leq \frac{\left(1-|\alpha|^{2}\right)^{n-1}}{|1-\langle z, \alpha\rangle|^{4(n-1)}} \beta^{n-1} .
$$

We conclude that

$$
\begin{aligned}
N\left(w ; f, \nu_{\alpha}\right) & <C\left(1-|\alpha|^{2}\right)^{n} \int_{f^{-1}(w)}\left(1-|z|^{2}\right) \beta^{n-1} \\
& <C\left(1-|\alpha|^{2}\right)^{n} N(w ; f, \sigma)
\end{aligned}
$$

with $C$ depending on $n$ and $\rho$.

Proof of Theorem 3. Under the hypotheses of the Theorem it suffices (recalling (15)) to show that for each fixed $\zeta \in \partial \mathbf{B}^{n}$ and $\rho>0$ that

$$
\lim _{\alpha \rightarrow \zeta} \int_{\partial \mathbf{B}^{n}}|f|^{2} d \nu_{\alpha}-|f(\alpha)|^{2} \leq \frac{1}{\pi} \operatorname{area}\left(f\left(D_{\rho, \zeta}\right)\right) .
$$

By the Hardy-Stein identity (10) this is equivalent to

$$
\lim _{\alpha \rightarrow \zeta} \int_{\Omega} N\left(w ; f, \nu_{\alpha}\right) d u d v \leq \frac{1}{2} \operatorname{area}\left(f\left(D_{\rho, \zeta}\right)\right) .
$$

Let $\zeta$ and $\rho$ be fixed and define $\Omega=f\left(\mathbf{B}^{n}\right)$ and $\Omega_{\rho}=f\left(D_{\rho, \zeta}\right)$. Let $C$ be the constant in (19) of Lemma 2 and define a function $h$ by

$$
h(w, \alpha)=C\left(1-|\alpha|^{2}\right)^{n} G_{\Omega}(w ; f(0))+G_{\Omega_{\rho}}(w ; f(\alpha)) .
$$

The Green function $G_{\Omega}$ is harmonic on $\Omega \backslash\{f(0)\}$, while $G_{\Omega_{\rho}}$ is harmonic on $\Omega_{\rho} \backslash\{f(\alpha)\}$ and 0 on $\Omega \backslash \bar{\Omega}_{\rho}$. It follows from Lemma 2 and the majorization principle (6) that $N\left(w ; f, \nu_{\alpha}\right) \leq h(w)$ on $\Omega \backslash \bar{\Omega}_{\rho}$. Since $h$ has a logarithmic pole at $f(\alpha)$ it now follows from the maximum principle that $N\left(w ; f, \nu_{\alpha}\right) \leq h(w)$ on $\bar{\Omega}_{\rho}$, and hence $N\left(w ; f, \nu_{\alpha}\right) \leq h(w)$ on all of $\Omega$.

We now have

$$
\begin{aligned}
\limsup _{\alpha \rightarrow \zeta} \int_{\Omega} N\left(w ; f, \nu_{\alpha}\right) d u d v & \leq \limsup _{\alpha \rightarrow \zeta} \int_{\Omega} h(w, \alpha) d u d v \\
& \leq \limsup _{\alpha \rightarrow \zeta} \int_{\Omega_{\rho}} G_{\Omega_{\rho}}(w, f(\alpha)) d u d v \\
& \leq \frac{1}{2} \operatorname{area}\left(\Omega_{\rho}\right) .
\end{aligned}
$$

This proves (21) as desired. 


\section{REFERENCES}

[1] H. Alexander, The area of the spectrum of an element of a uniform algebra, Complex Approximation, 3-12, Birkhauser, 1980.

[2] H. Alexander, B. A. Taylor, and J. Ullman, Areas of projections of analytic sets, Invent. Math., 16 (1972), 335-341.

[3] S. Axler and J. H. Shapiro, Putnam's theorem Alexander's spectral area estimate and $V M O$., (preprint).

[4] C. Bandle, Isoperimetric Inequalities and Applications, Pitman, 1980.

[5] M. Essen and D. F. Shea, On some questions of uniqueness in the theory of symmetrization, Ann. Acad. Sci. Fenn. Ser. A. I. Math., 4 (1978/79), 311-340.

[6] M. Essen, D. F. Shea, and C. Stanton, A value-distribution criterion for the class $L \log$ $L$ and some related question, Annales Inst. Fourier, (to appear).

[7] T. Gamelin, Uniform Algebras and Jensen Measures, London Math. Soc. Lecture Notes Series no. 32, Cambridge University Press, London and New York, 1979.

[8] P. A. Griffiths, Entire Holomorphic Mappings in One and Several Complex Variables, Princeton University Press, 1976.

[9] W. K. Hayman, Meromorphic Functions, Oxford University Press, 1964.

[10] O. Lehto, A majorant principle in the theory of functions, Math. Scand., 1 (1953), 5-17.

[11] G. Polya and G. Szego, Isoperimetric Inequalities in Mathematical Physics, Princeton University Press, Princeton, N. J., 1951.

[12] W. Rudin, Function Theory in the Unit Ball of $C^{n}$, Springer-Verlag, 1980.

[13] P. Stein, On a theory of M. Riesz, J. London Math. Soc., 8 (1933), 242-247.

[14] W. Stoll, Introduction to Value Distribution Theory of Meromorphic Maps, Lecture Notes in Mathematics no. 950, 210-359, Springer-Verlag, 1982.

Received June 6, 1985

UNIVERSITY OF CALIFORNIA, RIVERSIDE

RIVERSIDE, CA 92521 


\title{
PACIFIC JOURNAL OF MATHEMATICS \\ EDITORS
}

\author{
V. S. VARADARAJAN \\ (Managing Editor) \\ University of California \\ Los Angeles, CA 90024 \\ HERBERT CLEMENS \\ University of Utah \\ Salt Lake City, UT 84112 \\ R. FINN \\ Stanford University \\ Stanford, CA 94305
}

\author{
HERMANN FLASCHKA \\ University of Arizona \\ Tucson, AZ 85721
}

RAMESh A. GANGOLLI

University of Washington Seattle, WA 98195

VAUGHAN F. R. JONES

University of California

Berkeley, CA 94720

ROBION KIRBY

University of California

Berkeley, CA 94720
C. C. MOORE

University of California

Berkeley, CA 94720

H. SAMELSON

Stanford University

Stanford, CA 94305

HAROLD STARK

University of California, San Diego

La Jolla, CA 92093

\section{ASSOCIATE EDITORS}

\author{
R. ARENS \\ E. F. BECKENBACH \\ B. H. NeUmanN \\ F. WOLF \\ K. YOSHIDA \\ (1906-1982)

\section{SUPPORTING INSTITUTIONS} \\ UNIVERSITY OF ARIZONA \\ UNIVERSITY OF BRITISH COLUMBIA \\ CALIFORNIA INSTITUTE OF TECHNOLOGY \\ UNIVERSITY OF CALIFORNIA \\ MONTANA STATE UNIVERSITY \\ UNIVERSITY OF NEVADA, RENO \\ NEW MEXICO STATE UNIVERSITY \\ OREGON STATE UNIVERSITY \\ UNIVERSITY OF OREGON \\ UNIVERSITY OF SOUTHERN CALIFORNIA \\ STANFORD UNIVERSITY \\ UNIVERSITY OF HAWAII \\ UNIVERSITY OF TOKYO \\ UNIVERSITY OF UTAH \\ WASHINGTON STATE UNIVERSITY \\ UNIVERSITY OF WASHINGTON
}

The Supporting Institutions listed above contribute to the cost of publication of this Journal, but they are not owners or publishers and have no responsibility for its content or policies.

Mathematical papers intended for publication in the Pacific Journal of Mathematics should be in typed form or offset-reproduced (not dittoed), double spaced with large margins. Please do not use built up fractions in the text of the manuscript. However, you may use them in the displayed equations. Underline Greek letters in red, German in green, and script in blue. The first paragraph must be capable of being used separately as a synopsis of the entire paper. In particular it should contain no bibliographic references. Please propose a heading for the odd numbered pages of less than 35 characters. Manuscripts, in triplicate, may be sent to any one of the editors. Please classify according to the scheme of Math. Reviews, Index to Vol. 39. Supply name and address of author to whom proofs should be sent. All other communications should be addressed to the managing editor, or Elaine Barth, University of California, Los Angeles, California 90024.

There are page-charges associated with articles appearing in the Pacific Journal of Mathematics. These charges are expected to be paid by the author's University, Government Agency or Company. If the author or authors do not have access to such Institutional support these charges are waived. Single authors will receive 50 free reprints; joint authors will receive a total of 100 free reprints. Additional copies may be obtained at cost in multiples of 50 .

The Pacific Journal of Mathematics is issued monthly as of January 1966. Regular subscription rate: $\$ 190.00$ a year (5 Vols., 10 issues). Special rate: $\$ 95.00$ a year to individual members of supporting institutions.

Subscriptions, orders for numbers issued in the last three calendar years, and changes of address should be sent to Pacific Journal of Mathematics, P.O. Box 969, Carmel Valley, CA 93924, U.S.A. Old back numbers obtainable from Kraus Periodicals Co., Route 100, Millwood, NY 10546.

The Pacific Journal of Mathematics at P.O. Box 969, Carmel Valley, CA 93924 (ISSN 0030-8730) publish'es 5 volumes per year. Application to mail at Second-class postage rates is pending at Carmel Valley, California, and additional mailing offices. Postmaster: send address changes to Pacific Journal of Mathematics, P.O. Box 969, Carmel Valley, CA 93924.

PUBLISHED BY PACIFIC JOURNAL OF MATHEMATICS, A NON-PROFIT CORPORATION

Copyright (C) 1986 by Pacific Journal of Mathematics 


\section{Pacific Journal of Mathematics}

\section{Vol. 125, No. $2 \quad$ October, 1986}

Dale Edward Alspach, On $\mathscr{L}_{p, \lambda}$ spaces for small $\lambda \ldots \ldots \ldots \ldots \ldots \ldots 257$

Jong Sook Bae and Sangsuk Yie, Range of Gateaux differentiable operators

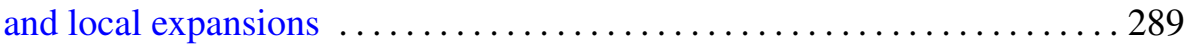

Hubert Berens and Lothar Hetzelt, On accretive operators on $l_{n}^{\infty} \ldots \ldots 301$

Marie-Françoise Bidaut-Véron, Global existence and uniqueness results for singular solutions of the capillarity equation ................. 317

Donald M. Davis and Mark Mahowald, Classification of the stable homotopy types of stunted real projective spaces ................. 335

Aad Dijksma, Heinz K. Langer and Hendrik S. V. de Snoo, Unitary

colligations in $\Pi_{\kappa}$-spaces, characteristic functions and Štraus extensions

Michel Enock and Jean-Marie Schwartz, Algèbres de Kac

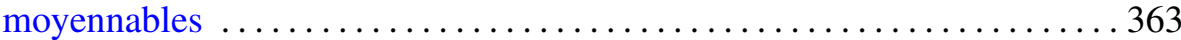

Seppo Granlund, Peter Lindqvist and Olli Martio, Note on the

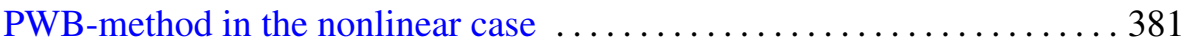

Palle E. T. Jorgensen, Analytic continuation of local representations of Lie

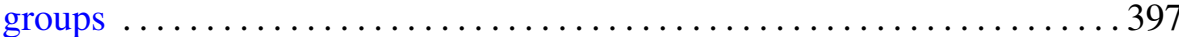

Robert P. Kaufman, Plane curves and removable sets ................409

José M. Montesinos and Wilbur Carrington Whitten, Constructions of two-fold branched covering spaces $\ldots \ldots \ldots \ldots \ldots$.

Benedict Seifert, Highly transitive group actions on trees and normalizing Tits systems

Charles Stuart Stanton, Counting functions and majorization for Jensen

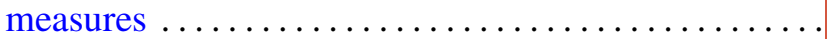

Luen-Fai Tam, On existence criteria for capillary free surfaces without gravity

Zhuocheng Yang, Exposed points of left invariant means 